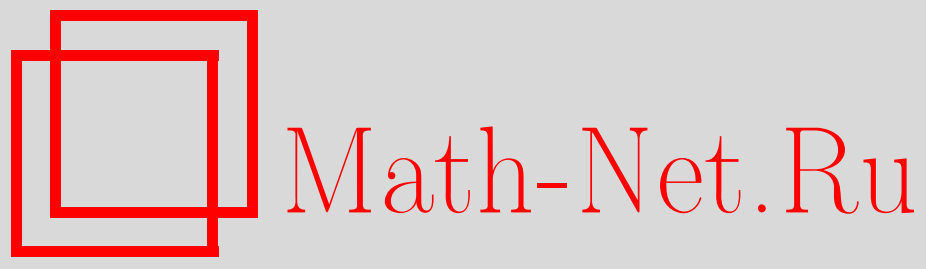

В. Н. Карпушкин, Об асимптотическом распределении спектра оператора по неприводимым представлениям его группы симметрий, Функи. анализ и его прил., 2006, том 40, выпуск 3, 72-75

DOI: https://doi.org/10.4213/faa746

Использование Общероссийского математического портала Math-Net.Ru подразумевает, что вы прочитали и согласны с пользовательским соглашением http://www. mathnet.ru/rus/agreement

Параметры загрузки:

IP : 54.157 .27 .8

26 апреля 2023 г., 14:48:53

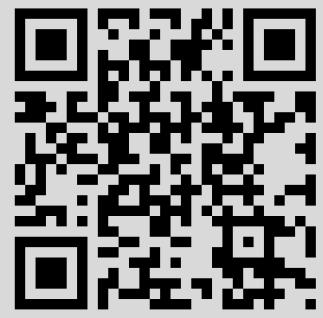


упрощения состоит в переходе от интегрирования по $\mathbb{Z}+i \mathbb{R}$ к интегрированию по двойственной группе $\mathbb{C}^{*}$. При этом ядра выражаются через общие гипергеометрические функции, связанные с грассманианом $G_{7,3}$. Ю. А. Неретин полагает, тем не менее, что указанный интеграл выражается через общеупотребительные функции. (Примечание при корректуре. Это предположение подтвердилось.)

Автор благодарит Ю. А. Неретина за ряд полезных замечаний и указание на работу [5].

\title{
ЛитературА
}

[1] П. И. Голод, Математические основы теории симметрий, Научн.-изд. центр «Регулярная и хаотическая динамика», Ижевск, 2001. [2] А. Ф. Никифоров, С. К. Суслов, В. Б. Уваров, Классические ортогоналъные полиномы дискретной переменной, Наука, М., 1985. [3] В. А. Кнып, П. П. Пипирайте, Ю. Ф. Смирнов, Ядерная физика, 22:5 (1975), 1063-1072. [4] М. А. Наймарк, Труды ММO, 5 (1959), 121-154. [5] W. Groenewelt, http://xxx.arxiv.org/abs/math.CA/0501511.

Московский государственный технический университет им. Н. Э. Баумана

Поступило в редакцию e-mail: ismagil@serv.bmstu.ru 24 сентября 2005 г.

\section{Об асимптотическом распределении спектра оператора по неприводимым представлениям его группы симметрий*}

\author{
(c) 2006. В. Н. КАРПУШКИН
}

В работе доказано, что асимптотически представление конечной группы в пространстве собственных функций эллиптического оператора на римановом многообразии, коммутирующего с эффективным действием рассматриваемой группы, кратно регулярному представлению этой группы.

1. Формулировка результата. Пусть $B$ - связное компактное $l$-мерное гладкое ориентируемое многообразие (быть может, с краем), на котором эффективно действует конечная группа $G$ из $m$ элементов (эффективность означает, что имеется орбита, состоящая из $m$ точек). Действие элемента $g \in G$ обозначим через $M(g)$. Зафиксируем $G$-инвариантную риманову метрику и соответствующую инвариантную меру $\mu$ на $B$.

Пусть $L_{2}(B)$ - пространство интегрируемых с квадратом по Лебегу функций на $B$. Действие группы $G$ на $B$ индуцирует представление $Q(g)$ этой группы в $L_{2}(B)$. В силу инвариантности меры это представление ортогонально.

Пусть группа $G$ имеет $n$ неприводимых вещественных неэквивалентных представлений. Обозначим через $\Gamma^{i}$ максимальное векторное подпространство функций из $L_{2}(B)$, преобразующихся по $i$-му неприводимому представлению.

Пусть $P$ есть $G$-инвариантный формально самосопряженный полуограниченный (снизу) эллиптический дифференциальный оператор четного порядка с гладкими коэффициентами на пространстве гладких функций на многообразии $B$, обращающихся в 0 в окрестности края $\partial B$. Пусть $\widetilde{P}-$ расширение

*Работа выполнена при поддержке гранта «Школа Арнольда». 
этого оператора по Фридрихсу (см. [10]). Для оператора $S$ обозначим через $N(S, \lambda)$ (соответственно через $\left.N^{i}(S, \lambda)\right)$ число собственных значений оператора $S$, меньших $\lambda$, с учетом кратности (соответственно тех из них, для которых собственные функции принадлежат $\left.\Gamma^{i}\right)$.

Долей $i$-го неприводимого представления группы $G$ в ее регулярном представлении (см. [8]) называется число $s_{i} d_{i} / m$, где $s_{i}-$ кратность $i$-го неприводимого представления в регулярном представлении, a $d_{i}-$ его размерность.

В комплексной ситуации $s_{i}=d_{i}$, а доля совпадает с мерой Планшереля (см. [2]).

Число $\lim _{\lambda \rightarrow+\infty} N^{i}(\widetilde{P}, \lambda) / N(\widetilde{P}, \lambda)$ (если такой предел существует) называется асимптотической долей $i$-го неприводимого представления группы $G$ в пространстве собственных функций оператора $\widetilde{P}$.

Пусть существует фундаментальная область $A$ группы $G$ на $B$ (см. [6]), состоящая из конечного числа компонент связности и измеримая по Жордану. Точнее, предположим, что существует $T<+\infty$ карт $\left(K_{t}, \varphi_{t}\right)$ на $B$, таких, что $\varphi_{t}: K_{t} \rightarrow \mathbb{R}^{l}, \bigcup_{t=1}^{T} K_{t} \supset A$ и $\nu\left(\varphi_{t}\left(\partial A \cap K_{t}\right)\right)=0$, где $\nu$ - мера Жордана в $\mathbb{R}^{l}$.

Теорема 1. Асимптотическая доля любого неприводимого представления группь $G$ в пространстве вещественнозначных собственных функиий оператора $\widetilde{P}$ существует и равна его доле в регулярном вещественном представлении группъ $G$.

Теорема 2. Асимптотическая доля любого неприводимого представления группы $G$ в пространстве комплекснозначных собственных функиий оператора $\widetilde{P}$ существует и равна его мере Планшереля.

Теоремы 1 и 2 эквивалентны. Поскольку доказательство основано на вещественных аргументах (вариационном принципе), мы будем доказывать теоремy 1.

2. Доказательство результата. Для открытого подмножества $U \subset B$ обозначим через $C_{0}^{\infty}(U)$ пространство гладких функций с носителем, содержащимся в $U$. Пусть $\lambda_{1} \leqslant \cdots \leqslant \lambda_{r} \leqslant \cdots-$ собственные значения оператора $\widetilde{P}$ с учетом кратности.

Из предположений о фундаментальной области $A$ следует, что если $B_{e}=$ $A \backslash \partial A$ и $B_{g}=M(g) B_{e}$, где $e-$ единица группы $G$, то подмножества $B_{g}$ многообразия $B$ открыты, измеримы по Жордану, состоят из конечного числа компонент связности, попарно не пересекаются и $\mu(B)=\mu\left(\bigcup_{g \in G} B_{g}\right)$.

ЛЕмма 1. Для любого $\varepsilon>0$ существуют открытые подмножества $B_{g}^{\varepsilon}$ множества $B$ с гладкими границами, такие, что замъкание подмножества $B_{g}^{\varepsilon}$ содержится в $B_{g}, \mu\left(B_{g}\right)>\mu\left(B_{g}^{\varepsilon}\right)>(1-\varepsilon) \mu\left(B_{g}\right) u M(g) B_{e}^{\varepsilon}=B_{g}^{\varepsilon}$ для всех $g \in G$.

ДокАзАтЕльство. Утверждение следует из предположений о фундаментальной области $A$.

Пусть $B^{\varepsilon}=\bigcup_{g \in G} B_{g}^{\varepsilon}, P_{\varepsilon}$ - сужение оператора $P$ на $C_{0}^{\infty}\left(B^{\varepsilon}\right)$ и $\widetilde{P}_{\varepsilon}-$ расширение по Фридрихсу оператора $P_{\varepsilon}$.

Лемма 2. В подпространстве собственных функиий оператора $\widetilde{P}_{\varepsilon}$, отвечающих произвольному собственному значению $\lambda$, существует (конечньй) базис 
$\left\{W_{l, g}\right\}, 1 \leqslant l \leqslant K_{\varepsilon}(\lambda), g \in G$, такой, что $W_{l, g}$ обращается в нуль вне замыкания множества $B_{g}^{\varepsilon}$ u $Q(g)\left(W_{l, e}\right)=W_{l, g}$ для всех $g \in G$ u всех $l, 1 \leqslant l \leqslant K_{\varepsilon}(\lambda)$.

ДокАзАтЕльство. Утверждение следует из вида асимптотики спектра (см. [9], [12]) (подпространство собственных функций, отвечающих собственному значению $\lambda$, конечномерно).

ЛЕмма 3. $N^{i}(\widetilde{P}, \lambda) \geqslant N^{i}\left(\widetilde{P}_{\varepsilon}, \lambda\right)$ для всех $\varepsilon>0,1 \leqslant i \leqslant n$.

ДокАЗАтЕльство. Задача на собственные значения для эллиптического оператора $\widetilde{P}$ (соответственно $\left.\widetilde{P}_{\varepsilon}\right)$ эквивалентна вариационной задаче в случае гладких границ $\partial B$ (соответственно $\left.\partial B^{\varepsilon}\right)$; см. [7], [9]. Сузим обе эти задачи на подпространство $\Gamma^{i}$. Тогда из условия $B^{\varepsilon} \subset B$ и из определения вариационной задачи следует, что $N^{i}(\widetilde{P}, \lambda) \geqslant N^{i}\left(\widetilde{P}_{\varepsilon}, \lambda\right)$ для всех $1 \leqslant i \leqslant n$.

ЛЕмма 4. Предположим, что для последовательности натуралъных чисел $q_{k}$, такой, что $q_{k} \rightarrow \infty$ при $k \rightarrow \infty$, существует предел

$$
\lim _{k \rightarrow \infty} N^{i}\left(\widetilde{P}, \lambda_{q_{k}}\right) / N\left(\widetilde{P}, \lambda_{q_{k}}\right) .
$$

Тогда этот предел не менъше, чем $s_{i} d_{i} / m$, при всех $1 \leqslant i \leqslant n$.

ДокАЗАТЕЛЬСтво. Положим $\lambda_{q_{k}}=r_{k}$. Заметим, что

$$
\frac{N^{i}\left(\widetilde{P}, r_{k}\right)}{N\left(\widetilde{P}, r_{k}\right)}=\frac{N^{i}\left(\widetilde{P}, r_{k}\right)}{N^{i}\left(\widetilde{P}_{\varepsilon}, r_{k}\right)} \frac{N^{i}\left(\widetilde{P}_{\varepsilon}, r_{k}\right)}{N\left(\widetilde{P}_{\varepsilon}, r_{k}\right)} \frac{N\left(\widetilde{P}_{\varepsilon}, r_{k}\right)}{N\left(\widetilde{P}, r_{k}\right)}
$$

Из леммы 3 вытекает, что $N^{i}\left(\widetilde{P}, r_{k}\right) \geqslant N^{i}\left(\widetilde{P}_{\varepsilon}, r_{k}\right)$. Из леммы 2 следует, что $N^{i}\left(\widetilde{P}_{\varepsilon}, r_{k}\right) / N\left(\widetilde{P}_{\varepsilon}, r_{k}\right)=s_{i} d_{i} / m$. Из работ [9], [12] и леммы 1 вытекает, что при $\lambda \rightarrow+\infty$ главный член асимптотики величины $N\left(\widetilde{P}_{\varepsilon}, \lambda\right)($ соответственно $N(\widetilde{P}, \lambda))$ есть $b_{\varepsilon} \lambda^{\sigma}$ (соответственно $b \lambda^{\sigma}$ ), причем $\sigma>0$ и $b_{\varepsilon} \rightarrow b$ при $\varepsilon \rightarrow 0$. Отсюда следует, что предел $\lim _{k \rightarrow \infty} N\left(\widetilde{P}_{\varepsilon}, r_{k}\right) / N\left(\widetilde{P}, r_{k}\right)$ существует и равен $1-\delta(\varepsilon)$, где $\delta(\varepsilon) \rightarrow 0$ при $\varepsilon \rightarrow 0$. Предел $\lim _{k \rightarrow \infty} N^{i}\left(\widetilde{P}, r_{k}\right) / N^{i}\left(\widetilde{P}_{\varepsilon}, r_{k}\right)$ существует и конечен при всех достаточно малых $\varepsilon>0$, поскольку существуют, конечны и не равны нулю пределы остальных дробей в равенстве (1).

Поскольку $\varepsilon>0$ произвольно, получаем лемму 4.

Заметим, что $\sum_{i=1}^{n} N^{i}(\widetilde{P}, \lambda)=N(\widetilde{P}, \lambda)$, так как всякое подпространство, отвечающее какому-либо собственному значению, инвариантно и конечномерно ([9], [12]), а всякое инвариантное конечномерное подпространство разлагается в прямую сумму неприводимых.

Предположим, что существует $j, 1 \leqslant j \leqslant n$, и последовательность $q_{k}$, такая, что $\lim _{k \rightarrow \infty} N^{j}\left(\widetilde{P}, r_{k}\right) / N\left(\widetilde{P}, r_{k}\right)>s_{j} d_{j} / m$, где $r_{k}=\lambda_{q_{k}}$. Выберем из нее подпоследовательность $q_{k_{l}}$ так, чтобы для всех $i, 1 \leqslant i \leqslant n$, существовали пределы $\lim _{l \rightarrow \infty} N^{i}\left(\widetilde{P}, \eta_{l}\right) / N\left(\widetilde{P}, \eta_{l}\right)$, где $\eta_{l}=r_{k_{l}}$. Тогда в силу леммы 4 получим противоречие, так как $\sum_{i=1}^{n} s_{i} d_{i} / m=1$ и сумма этих пределов равна $1 . \mathrm{Ta-}$ ким образом, предельные точки последовательностей $N^{i}\left(\widetilde{P}, \lambda_{q}\right) / N\left(\widetilde{P}, \lambda_{q}\right)$ для каждого $i, 1 \leqslant i \leqslant n$, единственны и равны $s_{i} d_{i} / m$. Теорема доказана.

ГиПотЕзА. Для любого эффективного действия конечной группь на компактном многообразии существует фундаментальная область, измеримая по Жордану и состоящая из конечного числа компонент связности. 
Настоящая работа возникла из обдумывания гипотезы В. И. Арнольда о «наиболее вероятных» представлениях (см. [1]). Попытка доказательства этой гипотезы содержится в работе [5]. К сожалению, в этом доказательстве имеются пробелы.

Автор благодарен В. И. Арнольду за постановку задачи и внимание к работе, М. С. Аграновичу, С. М. Гусейн-Заде и А. В. Чернавскому за полезные обсуждения.

Автор благодарен рецензенту за указание работ [3], [4], [11], в которых подобный результат получен другими методами.

\title{
ЛитеРАТУРА
}

[1] В. И. Арнольд, Задачи семинара 2003-2004, МЦНМО, М., 2005. [2] А. М. Вершик, С. В. Керов, Функи. анализ и его прил., 19:1 (1985), 25-36. [3] H. Donnelly, Math. Ann., 224 (1976), 161-170. [4] H. Donnelly, Math. Ann., 237 (1978), 23-40. [5] В. Н. Карпушкин, Вестник МГУ, матем., мех., 1974, № 2, 9-13. [6] Ф. Клейн, Лекиии о развитии математики в ХIX столетии, Наука, М., 1989. [7] Р. Курант, Д. Гильберт, Методы математической физики, т. 1, Гостехиздат, М., 1951. [8] Г. Е. Любарский, Теория групn и ее применение в физике, М., 1958. [9] К. Морен, Методы гильбертова пространства, Мир, М., 1965. [10] Ф. Рисс, Б. Секефальви-Надь, Лекиии по функииональному анализу, Мир, М., 1979. [11] B. Helffer, D. Robert, Amer. J. Math., 106 (1984), 1199-1236. [12] М. А. Шубин, Псевдодифференциальные операторы и спектральная теория, Наука, М., 1978.

Институт проблем передачи информации РАН e-mail: vladkarp@rol.ru

Поступило в редакцию 16 февраля 2005 г.

УДК $512.662 .1+512.818 .4$

\section{Деформации бесконечномерной алгебры Ли $L_{3}{ }^{*}$}

\author{
(C) 2006. Ю. Ю. Кочетков
}

1. Введение. Обозначим через $W$ алгебру Ли полиномиальных векторных полей на прямой с базисом $e_{i}=x^{i+1} \frac{d}{d x}, i=-1,0, \ldots,\left[e_{i}, e_{j}\right]=(j-i) e_{i+j}$. Алгебра $W$ градуирована: $\operatorname{deg}\left(e_{i}\right)=i$. Через $L_{k} \subset W, k>0$, обозначим нильпотентную подалгебру, порожденную элементами $e_{i}, i \geqslant k$.

Деформации алгебр $L_{1}$ и $L_{2}$ активно изучались. В работах [1] и [2] были найдены инфинитезимальные деформации и вычислены квадраты Масси для алгебр $L_{1}$ и $L_{2}$, а также был решен вопрос о продолжаемости однородных инфинитезимальных деформаций до глобальных. В работе [3] был предъявлен полный список попарно не эквивалентных деформаций алгебры $L_{1}$, в том числе была найдена деформация, которой не отвечает никакая инфинитезимальная деформация (соответствующий 2-коцикл нулевой). В работе [4] была определена миниверсальная деформация произвольной алгебры Ли и предложена схема построения версальной деформации. Используя этот аппарат, авторы провели

*Эта работа была поддержана РФФИ, проект №04-01-00647. 